\title{
UPDATE ARTICLE
}

\section{Stereotyped movement disorder in ICD-11}

\author{
Dan J. Stein, ${ }^{1}$ Douglas W. Woods ${ }^{2}$ \\ ${ }^{1}$ Department of Psychiatry, University of Cape Town, Cape Town, South Africa. ${ }^{2}$ Psychology Department, Texas A\&M University, \\ College Station, TX, USA.
}

\begin{abstract}
According to current proposals for ICD-11, stereotyped movement disorder will be classified in the grouping of neurodevelopmental disorders, with a qualifier to indicate whether self-injury is present, similar to the classification of stereotypic movement disorder in DSM-5. At the same time, the WHO ICD-11 Working Group on the Classification of Obsessive-Compulsive and Related Disorders has proposed a grouping of body-focused repetitive behavior disorders within the obsessive-compulsive and related disorders (OCRD) cluster to include trichotillomania and skin-picking disorder. DSM-5 has taken a slightly different approach: trichotillomania and excoriation (skin picking) disorder are included in the OCRD grouping, while body-focused repetitive behavior disorder is listed under other specified forms of OCRD. DSM-5 also includes a separate category of nonsuicidal self-injury in the section on "conditions for further study." There are a number of unresolved nosological questions regarding the relationships among stereotyped movement disorder, body-focused repetitive behavior disorders, and nonsuicidal self-injury. In this article, we attempt to provide preliminary answers to some of these questions as they relate to the ICD-11 classification of mental and behavioral disorders.
\end{abstract}

Keywords: ICD-11; DSM-5; classification; stereotyped movement disorder; stereotypic movement disorder; obsessive-compulsive and related disorders; body-focused repetitive behavior disorder

\section{Introduction}

There are several areas of scientific literature relevant to stereotyped/stereotypic movement disorder (SMD). First, the literature on intellectual disability and pervasive developmental disorders has noted that SMD is prevalent in individuals with these conditions, and can be an important target of treatment. ${ }^{1,2}$ Second, the pediatric neurology literature has emphasized that SMD may present in children without intellectual disability or pervasive development disorders, and require appropriate management., ${ }^{3,4}$ Third, a number of papers have focused on body-focused repetitive behaviors or on nonsuicidal self-injurious behavior in patients with normal development. ${ }^{5,6}$ Given WHO's current revision of the ICD-10, it is timely to establish a synthesis of these different literatures so that nosological questions in the field can be fully clarified. This brief paper raises a number of these questions, and provides preliminary answers relevant to the development of the ICD-11 classification of mental and behavioral disorders.

\section{SMD in ICD-10 and DSM-5}

In ICD-10, stereotyped movement disorders (SMDs) appear in the grouping of "behavioral and emotional disorders with onset usually occurring in childhood and adolescence" (F90-F98). They are described as being

Correspondence: Dan J. Stein, Department of Psychiatry, University of Cape Town and Groote Schuur Hospital, J-Block, Anzio Road, Observatory 7925, South Africa.

E-mail: dan.stein@uct.ac.za characterized by voluntary, repetitive, stereotyped, nonfunctional (and often rhythmic) movements that do not form part of any recognized psychiatric or neurological condition. ICD-10 emphasizes that when such movements occur as symptoms of some other disorder, such as a pervasive developmental disorder, only the overall disorder should be coded. However, when mental retardation occurs together with SMD, both disorders should be coded. SMD movements that are non-injurious include body-rocking, head-rocking, hair-plucking, hairtwisting, finger-flicking mannerisms, and hand-flapping. Self-injurious stereotyped behavior includes repetitive head-banging, face-slapping, eye-poking, and biting of hands, lips, or other body parts. Nail-biting, thumbsucking, and nose-picking, as well as trichotillomania, are specifically excluded. The ICD-10 section on pervasive developmental disorders also includes the diagnosis of "overactive disorder associated with mental retardation and stereotyped movements."

In DSM-5, stereotypic movement disorder (SMD) is included in the neurodevelopmental section, where the diagnostic criteria indicate that it is characterized by repetitive, seemingly driven, and apparently purposeless motor behavior, with onset in the early developmental period, that interferes with activities and that may result in self-injury. DSM-5 requires that the behavior not be better explained by another neurodevelopmental or mental disorder, such as obsessive-compulsive disorder or trichotillomania, and not be attributable to the physiological effects of a substance or neurological condition. Specifiers of self-injurious behavior and severity are provided. Confusingly, given the exclusion of neurodevelopmental disorders, a qualifier is also provided for association with a 
known medical or genetic condition, neurodevelopmental disorder, or environmental factor.

DSM-5 also includes two other conditions relevant to a consideration of SMD. First, in the chapter on obsessivecompulsive and related disorders (OCRD), the diagnosis of other specified OCRD includes the term body-focused repetitive behavior disorder. This is characterized by recurrent body-focused repetitive behaviors (e.g., nailbiting, lip-biting, cheek-chewing) and repeated attempts to decrease or stop the behaviors. DSM- 5 emphasizes that these symptoms cause clinically significant distress or impairment and are not better explained by conditions such as trichotillomania, excoriation disorder, SMD, or nonsuicidal self-injury.

Second, the section of DSM-5 on "conditions for further study" includes nonsuicidal self-injury. The diagnostic criteria for nonsuicidal self-injury include engagement in intentional self-inflicted damage to the surface of one's body, of a sort likely to induce bleeding, bruising, or pain, with the expectation that the injury will lead to only minor or moderate physical harm. The absence of suicidal intent is either reported by the patient or can be deduced by frequent use of methods that the patient knows, or has learned, not to have lethal potential. The behavior is not socially sanctioned, and is not restricted to picking a scab or nail-biting. DSM-5 notes that the intentional self-injury aims to obtain relief from negative feelings or thoughts, to resolve an interpersonal difficulty, or to induce a positive feeling. Furthermore, the intentional self-injury is associated with negative feelings or thoughts or interpersonal difficulties, preoccupation with the intended behavior that is difficult to resist, and frequent thinking about selfinjury. The behavior or its consequences cause clinically significant distress or impairment. Furthermore, the behavior does not occur exclusively during states of psychosis, delirium, or intoxication, is not part of a pattern of repetitive stereotypies in individuals with a developmental disorder, and cannot be accounted for by another mental or medical disorder.

\section{SMD in ICD-11}

ICD-11 aims to have clinical utility, global applicability, and provide a version that is suitable for use in primary care settings by non-specialist clinicians. Given these aims, how should the section on SMD be updated? We raise three questions, and provide preliminary answers to each.

\section{1) In which grouping should SMD be classified?}

As noted, ICD-10 places SMD in the section on "other behavioral and emotional disorders with onset usually occurring in childhood and adolescence." Notably, the WHO ICD-11 Working Group on the Classification of Obsessive-Compulsive and Related Disorders has proposed a grouping of body-focused repetitive behavior disorders, which includes trichotillomania and skin-picking disorder, given the phenomenological and psychobiological overlap between these conditions and the potential clinical utility of conceptualizing them as closely related. ${ }^{7,8}$
Some authors have suggested that SMD should be a single entity, which includes both stereotypic behaviors in those with intellectual disability (e.g., head-banging) and body-focused repetitive behaviors (e.g., hair-pulling, skinpicking, cheek-chewing, lip-biting) in those without intellectual disability, and that this broader construct should be included in the chapter on OCRD, alongside tic disorders. ${ }^{9}$ This view is consistent with the hypothesis that stereotypies in a range of disorders have overlaps in underlying psychobiology. ${ }^{10,11}$ According to this line of thinking, stereotypies such as head-banging and cheekchewing ${ }^{12}$ could then be classified alongside trichotillomania and skin-picking disorder.

However, the literatures on stereotyped behavior in intellectual disability and pervasive developmental disorder (e.g., head-banging) and in individuals of normal intelligence seen in pediatric neurology clinics (e.g., hand-flapping) and in OCRD clinics (e.g., hair-pulling) have evolved separately, and there is perhaps insufficient data to make the case that a single entity would have clinical utility or diagnostic validity. Nevertheless, if there is to be an ICD-11 diagnosis of SMD in the neurodevelopmental section, and a grouping of body-focused repetitive behavior disorders in the section on OCRD, then it is important to provide information that helps clinicians to make the differential diagnosis. One possibility, consistent with that taken in DSM-5, is to emphasize that SMD begins very early in life; this is consistent with the literature on SMD in patients with intellectual disability and pervasive developmental disorders, as well as with the pediatric neurology literature on SMD in patients with normal development.

\section{2) What is the appropriate diagnostic hierarchy for SMD?}

A first question is whether SMD should be diagnosed in the presence of autistic disorder or neurogenetic conditions such as Lesch-Nyhan syndrome or Prader-Willi syndrome. Clearly, SMD can be a focus of management in such conditions. ICD-10 supports the hierarchy rule, indicating that SMD should not be diagnosed in such patients. DSM-5, on the one hand, has the medical exclusion criterion (which indicates that SMD should not be diagnosed when better explained by other neurological, mental, or neurodevelopmental conditions), but, on the other hand, provides a medical association specifier (when SMD is associated with a known medical, genetic, or neurodevelopmental condition).

The view of the WHO ICD-11 Working Group on the Classification of Obsessive-Compulsive and Related Disorders is that SMD should be able to be diagnosed as an independent condition in a range of medical conditions, such as Lesch-Nyhan syndrome and PraderWilli syndrome. This would help maximize the clinical utility of the nosology, and would help encourage research on SMD in such conditions. In fact, the literature on these conditions frequently refers to SMD as an important target of treatment.

In patients with autistic disorder, repetitive behaviors are common and diagnostic. Nevertheless, they may 
again be characterized by specific neurobiological alterations, and they may constitute a particular treatment target. ${ }^{13}$ One approach here may therefore be to have a specifier for SMD that acknowledges when it is secondary to a pervasive developmental disorder. Such a specifier would, on the one hand, emphasize that the SMD is not the primary diagnosis, but would, on the other hand, encourage clinicians to think about treatments that emerge from the general literature on SMD.

\section{3) How to differentiate SMD from body-focused repetitive} behavior disorders and nonsuicidal self-injury?

As alluded to earlier, there may in fact be considerable phenomenological overlap between SMD, trichotillomania, skin-picking disorder, and nonsuicidal self-injury. ICD-10 seems to indicate that in SMD there may be skinplucking, while in trichotillomania there is hair-pulling; in the clinic, it is not clear that such a differentiation exists. DSM-5 indicates that nonsuicidal self-injury should not be used for picking of scabs, but, in other respects, the DSM5 description of nonsuicidal self-injury has a good deal in common with that of excoriation (skin picking) disorder. ${ }^{14,15}$ As suggested earlier, as there is little overlap between the literature on SMD in intellectual disability and pervasive developmental disorders (often characterized by self-injury) and in pediatric neurology (typically without self-injury) and the literature on body-focused repetitive behavior disorders in patients with normal development. resulting in insufficient data on the extent of the overlap of these conditions, it may be appropriate to maintain the distinction between SMD (among the neurodevelopmental disorders) and body-focused repetitive behavior disorders (in the OCRD grouping).

However, our view is that there is significant evidence of an overlap between hair-pulling, skin-picking, and a range of other body-focused repetitive behaviors (e.g., cheek-chewing) in individuals without intellectual disability, often with onset at puberty or later. While trichotillomania and skin-picking disorder are the most prevalent and best characterized of these conditions, patients with any one of these conditions are at significant risk of suffering from any one of the others, ${ }^{16,17}$ and they respond to similar treatments. ${ }^{18}$ Thus, the Working Group has proposed for ICD-11 a broad construct of body-focused repetitive behavior disorders, with trichotillomania and skin-picking disorders as exemplars, but allowing also classification of those individuals with normal development and childhood/adolescent onset of other body-focused repetitive behaviors, such as cheekchewing. This would encourage clinicians to screen for a range of these disorders and to be aware of their overlap in symptomatology, but at the same time to be cognizant of the particular importance of trichotillomania and skinpicking disorder as more prevalent disorders for which there are well-studied treatment options.

The WHO ICD-11 Working Group on the Classification of Obsessive-Compulsive and Related Disorders is not, however, persuaded that there is sufficient data to differentiate body-focused repetitive behavior disorders and nonsuicidal self-injury. (Note that the developers of DSM-5 were also not completely persuaded of the validity of the nonsuicidal self-injury category, hence its placement in the section on "conditions for further study.") One possible distinction, alluded to in DSM-5, is that nonsuicidal self-injury is characterized by the specific intent to harm the body. However, this distinction requires further investigation, as both body-focused repetitive behavior disorders and nonsuicidal self-injury are characterized by repetitive self-injurious behavior. Both conditions often feature preceding tension and subsequent gratification, although this is not universally present. ${ }^{19}$ Much more needs to be done to understand the psychobiology of these disorders before a compelling argument that they are similar (or dissimilar) can be made more comprehensively. However, it is notable that the evaluation and treatment of patients with repetitive self-injurious behavior is currently quite similar, irrespective of the particular nature of the habitual self-injurious behavior. ${ }^{20,21}$

\section{Conclusion}

This article has emphasized that there are several different literatures relevant to SMD and to self-injury, ranging from work on self-injurious behavior in individuals with intellectual disability and pervasive developmental disorders to work on body-focused repetitive behavior and body-focused repetitive behavior disorder in individuals with OCRD. It is conceivable that these terms all describe the same entity, with non-substantive distinctions primarily reflecting differences in developmental trajectory and treatment setting. However, it is also quite possible that there is significant heterogeneity between different patients with stereotypic behaviors, and that multiple different diagnoses should be established. ${ }^{22}$

The WHO ICD-11 Working Group on the Classification of Obsessive-Compulsive and Related Disorders has argued for a compromise position, consistent with much previous work. In particular, maintenance of the distinction between SMD and body-focused repetitive behavior disorders is recommended. Within SMD, there may be a distinction between patients with intellectual disability or pervasive developmental disorders together with selfinjurious behavior, and those who present in pediatric neurology without developmental disorders or self-injurious behavior. The Working Group notes that SMD may be secondary to medical/genetic disorders and emphasizes the potential value of a SMD specifier that also allows for this diagnosis in patients with pervasive developmental disorders. Within the body-focused repetitive behavior disorders, trichotillomania and skin-picking disorder are the most prevalent conditions, but the Working Group has recommended a grouping of body-focused repetitive behavior disorder to facilitate the recognition of other forms of repetitive or self-injurious behavior.

\section{Acknowledgements}

The Department of Mental Health and Substance Abuse, World Health Organization, has received direct support 
that contributed to the activities of the Working Group from several sources: the International Union of Psychological Science, the National Institute of Mental Health (USA), the World Psychiatric Association, the Spanish Foundation of Psychiatry and Mental Health (Spain), and the Santander Bank UAM/UNAM endowed Chair for Psychiatry (Spain/Mexico).

DJS is supported by the Medical Research Council of South Africa.

\section{Disclosure}

DJS and DWW are members of the WHO ICD-11 Working Group on the Classification of ObsessiveCompulsive and Related Disorders, reporting to the International Advisory Group for the Revision of ICD-10 Mental and Behavioural Disorders. Unless specifically stated, the views expressed in this article are those of the authors and do not represent the official policies or positions of the Working Group, of the International Advisory Group, or of the WHO.

DJS has received research grants and/or consultancy honoraria from AMBRF, Biocodex, Cipla, Lundbeck, the National Responsible Gambling Foundation, Novartis, Servier, and Sun. DWW receives book royalties from Guilford Press, Springer Press, and Oxford University Press.

\section{References}

1 Lanovaz MJ. Towards a comprehensive model of stereotypy: integrating operant and neurobiological interpretations. Res Dev Disabil. 2011;32:447-55

2 Stein DJ, Garner JP, Keuthen NJ, Franklin ME, Walkup JT, Woods DW. Trichotillomania, stereotypic movement disorder, and related disorders. Curr Psychiatry Rep. 2007;9:301-2.

3 Singer HS. Stereotypic movement disorders. Handb Clin Neurol. 2011:100:631-9.

4 Freeman RD, Soltanifar A, Baer S. Stereotypic movement disorder: easily missed. Dev Med Child Neurol. 2010;52:733-8.

5 Castellanos FX, Ritchie GF, Marsh WL, Rapoport JL. DSM-IV stereotypic movement disorder: persistence of stereotypies of infancy in intellectually normal adolescents and adults. J Clin Psychiatry. 1996;57:116-22.

6 In-Albon T, Ruf C, Schmid M. Proposed diagnostic criteria for the DSM-5 of nonsuicidal self-injury in female adolescents: diagnostic and clinical correlates. Psychiatry J. 2013;2013:159208.
7 Lochner C, Simeon D, Niehaus DJ, Stein DJ. Trichotillomania and skin-picking: a phenomenological comparison. Depress Anxiety. 2002;15:83-6.

8 Snorrason I, Belleau EL, Woods DW. How related are hair pulling disorder (trichotillomania) and skin picking disorder? A review of evidence for comorbidity, similarities and shared etiology. Clin Psychol Rev. 2012;32:618-29.

9 Stein DJ, Grant JE, Franklin ME, Keuthen N, Lochner C, Singer HS, et al. Trichotillomania (hair pulling disorder), skin picking disorder, and stereotypic movement disorder: toward DSM-V. Depress Anxiety. 2010;27:611-26.

10 Ridley RM. The psychology of perserverative and stereotyped behaviour. Prog Neurobiol. 1994;44:221-31.

11 Stein DJ, Chamberlain SR, Fineberg N. An A-B-C model of habit disorders: hair-pulling, skin-picking, and other stereotypic conditions. CNS Spectr. 2006;11:824-7.

12 Sarkhel S, Praharaj SK, Akhtar S. Cheek-biting disorder: another stereotypic movement disorder? J Anxiety Disord. 2011;25: 1085-6.

13 Hollander E, Soorya L, Chaplin W, Anagnostou E, Taylor BP, Ferretti $\mathrm{CJ}$, et al. A double-blind placebo-controlled trial of fluoxetine for repetitive behaviors and global severity in adult autism spectrum disorders. Am J Psychiatry. 2012;169:292-9.

14 Snorrason I, Stein DJ, Woods DW. Classification of excoriation (skin picking) disorder: current status and future directions. Acta Psychiatr Scand. 2013;128:406-7.

15 Grant JE, Odlaug BL, Chamberlain SR, Keuthen NJ, Lochner C, Stein DJ. Skin picking disorder. Am J Psychiatry. 2012;169:1143-9.

16 Stein DJ, Flessner CA, Franklin M, Keuthen NJ, Lochner C, Woods DW. Is trichotillomania a stereotypic movement disorder? An analysis of body-focused repetitive behaviors in people with hairpulling. Ann Clin Psychiatry. 2008;20:194-8.

17 Snorrason I, Ricketts EJ, Flessner CA, Franklin ME, Stein DJ, Woods DW. Skin picking disorder is associated with other bodyfocused repetitive behaviors: findings from an internet study. Ann Clin Psychiatry. 2012;24:292-9.

18 Bate KS, Malouff JM, Thorsteinsson ET, Bhullar N. The efficacy of habit reversal therapy for tics, habit disorders, and stuttering: a metaanalytic review. Clin Psychol Rev. 2011;31:865-71.

19 Lochner C, Stein DJ, Woods D, Pauls DL, Franklin ME, Loerke EH, et al. The validity of DSM-IV-TR criteria $B$ and $C$ of hair-pulling disorder (trichotillomania): evidence from a clinical study. Psychiatry Res. 2011;189:276-80.

20 Woods DW, Wetterneck CT, Flessner CA. A controlled evaluation of acceptance and commitment therapy plus habit reversal for trichotillomania. Behav Res Ther. 2006;44:639-56.

21 Flessner CA, Busch AM, Heideman PW, Woods DW. Acceptanceenhanced behavior therapy (AEBT) for trichotillomania and chronic skin picking: exploring the effects of component sequencing. Behav Modif. 2008:32:579-94.

22 Freeman R. Stereotypic movement disorder: a case for Procrustes? Dev Med Child Neurol. 2014 Jul 9. [Epub ahead of print] 